\title{
Predictive Landing Guidance in Synthetic Vision Displays
}

\author{
R.R.D. Arents ${ }^{1}$, J. Groeneweg ${ }^{1}$, C. Borst ${ }^{2}$, M.M. van Paassen ${ }^{2}$ and M. Mulder ${ }^{*}, 2$ \\ ${ }^{I}$ National Aerospace Laboratory NLR, Cockpit \& Flight Operations (ATCF) Department, The Netherlands \\ ${ }^{2}$ Delft University of Technology, Faculty of Aerospace Engineering, Control and Simulation Section, The Netherlands
}

\begin{abstract}
Pilot manual path-following performance with synthetic vision displays can be improved with predictive guidance symbols. Little is known, however, on how these predictive guidance concepts can be applied to the landing flare maneuver. This paper discusses the applicability of 3D predictive guidance in synthetic vision displays during the final phase of the landing. Two types of predictive guidance were examined, the Flight-Path Predictor that indicates the aircraft's future position a certain time ahead, and the Flight Trajectory Predictor that presents the future trajectory by interpolating a number of sequential predicted positions. A theoretical investigation and an offline simulation were used to optimize the two guidance laws for the manual landing task. A pilot-in-the-loop experiment, conducted in a moving-base flight simulator, indicated that both predictive guidance types investigated support pilots in manual control. The pilot's ability to determine the correct flare initiation time is improved, and becomes comparable to timing the flare with a more realistic synthetic vision display with textured surfaces. Even though the flare initiation timing was improved by the addition of predictive guidance, the control of the flare after its initiation was not sufficiently supported. As a result, no noticeable improvement in landing performance was found.
\end{abstract}

Keywords: perspective flight-path displays, landing guidance, pilot performance, synthetic vision displays.

\section{INTRODUCTION}

Especially in general aviation, Synthetic Vision Systems (SVS) start to find their way into the cockpit. In an SVS, a synthetic computer-generated image of the world outside the cockpit is presented on a synthetic vision display, considerably improving a pilot's situation awareness. The artificial view of the outside world, restoring the visual contact with the ground at all times, can be further augmented by presenting the reference trajectory to be followed in 3D perspective, yielding a perspective flight-path display also known as a 'tunnel-in-thesky' [1-3]. As these smaller aircraft are generally not equipped with advanced automatic landing systems, the autoland system, most of the approaches and landings are conducted by the pilot in full manual control of the aircraft motion relative to the target runway.

Surprisingly little research has been conducted on whether synthetic vision displays, including the perspective flight-path presentation, can support pilots in manual control during the final phase of the landing. Whereas the navigation and path-following performance has been shown to improve during approach and final approach, the actual landing of the aircraft, i.e., the initiation and control of the flare, has rarely been studied. Given the often poor performance of pilots when landing in a simulator, the landing performance with a comparable artificial instrument, the synthetic vision display, may also be much worse as compared to landing using the natural out-of-the-window visual array. Issues such as the

*Address correspondence to this author at the Delft University of Technology, Faculty of Aerospace Engineering, Control and Simulation Section, The Netherlands; Tel: +31 15278 2094; Fax: +31 15278 6480;

E-mail: M.Mulder@tudelft.nl relatively small size of the synthetic display, the availability and quality of its textures, the choice of its field of view and related issues of 'conformal angles' of the presentation might play a role here. The possible effects of safety of mediocre or even bad pilot performance, especially in single pilot operations with small general aviation aircraft, are clear motivations to study pilot landing performance with synthetic instruments.

In the development of the perspective flight-path display in the 1980s and 1990s, various display augmentation principles have been developed to improve path-following accuracy [1-5]. The main example is the flight-path predictor (FPP), showing the future position of the aircraft a few seconds ahead in time. This 'predictive' display augmentation principle has shown to be very successful in improving manual path-following performance; however, there has been relatively little attention for the landing phase.

In this paper we investigate the landing performance of pilots using a synthetic vision display, and also study whether the use of predictive landing guidance can be used to improve performance. The synthetic vision displays studied include a perspective projection of the flight path. Two predictive guidance concepts will be discussed, the above-mentioned flight-path predictor, and the flighttrajectory predictor discussed later. In particular the pilot performance in initiating and controlling the flare will be investigated.

The paper is structured as follows. First, the landing flare is discussed in detail, including a summary of its sub-phases, the maneuver itself, and possible strategies to initiate and control the flare. Secondly, the two predictive guidance concepts will be discussed followed by a description of how 
these guidance laws were tuned in off-line simulations. Third, the pilot-in-the-loop experiment that has been conducted to investigate both concepts will be discussed. The results of the experiment, conducted in a moving-base simulator, are discussed next, followed by conclusions and recommendations.

\section{PHASES OF THE LANDING}

The final phase of the landing can generally be broken down in four sub-phases [6]; the approach, the flare, the touchdown control, and the landing roll. These sub-phases are not always distinguishable during every landing, but still provide a good reference for analysis. Here each sub-phase will be described briefly, followed by a more elaborated description of the flare maneuver in the next section.

"A good landing starts with a good approach", an old saying that indicates the importance of the first sub-phase. This sub-phase starts when the aircraft intercepts the glide path. The glide path is a (straight) fictitious line that is parallel to the runway, has a vertical declination of approximately 3 degrees, and connects to the touchdown zone on the runway. The (auto) pilot continuously controls the aircraft to follow the glide path after interception, while changes in lift, drag, thrust, weight, crosswind, headwind or atmospheric disturbances cause it to deviate it from its intended path.

The descent speed during the approach depends on the airspeed and the glide path's slope. Generally, the descent velocity is too high to allow smooth touchdown, and should be decreased for passenger comfort and landing gear impact tolerance. Decreasing the velocity of the descent is the goal of the second sub-phase, the flare maneuver. The flare is essentially a pull-up maneuver that increases the angle of attack, resulting in an increase of lift. Consequently, the descent velocity and the landing loads at touchdown are reduced. This sub-phase is discussed more thoroughly in the following section because of its importance to this study.

In the ideal case, the touchdown should occur immediately after the completion of the flare. For this ideal case, the touchdown control sub-phase is not distinguishable and the landing roll will follow immediately after the flare. Nevertheless, sometimes the aircraft is still above the runway after completion of the flare, and the touchdown control component of the landing will assure that touchdown eventually occurs. During touchdown control the aircraft generally consumes large amounts of runway length, which should be prevented.

The first contact with the runway pavement should occur at the main landing gear, which is designed to absorb most of the vertical kinetic energy. As the aircraft rolls over the runway during the landing roll sub-phase, it starts decelerating and the positive pitch angle will decrease until the nose-wheel gently touches the ground. The landing is still not completed. Remaining is the essential task of further decelerating the aircraft to normal taxi speed or a complete stop. This completes the final phase of the landing.

\section{THE FLARE MANEUVER}

The final part of the landing is essentially a sequence of two straight trajectories. During the approach sub-phase, the pilot follows three-degree glide path and after touchdown the aircraft rolls along the horizontal runway pavement. Just before touchdown, the (auto-) pilot executes a flare maneuver which takes care of the transition between two straight trajectories and allows a smooth landing. This transition is initiated at the flare initiation height by gradually increasing the elevator deflection and exciting the phugoid eigenmotion of the aircraft.

In case the autopilot is engaged during the flare, the autoland system generally executes a pre-programmed pitch rate command profile that depends on the descent velocity and starts at a predetermined initiation height until the aircraft touches the runway [7]. More advanced autoland systems also control thrust, brakes and spoilers to reduce total velocity.

Clearly, one of the main differences between automatic and manual landings is the greater consistency of the first [8]. The variation in the vertical velocity at touchdown and touchdown point dispersion, two important factors that determine the success of the landing maneuver, are smaller for automatic landings. The higher consistency also poses some challenges, however, such as runway wear due to hitting it on the same spot every landing. In fact, some automatic landing systems have been adapted to introduce some (deliberate) inconsistency.

The manual flare is performed in more or less the same way as described above for the autoland systems. Although it is possible to perform the flare with the steering column as single control, using the throttle as secondary control offers a better performance in the presence of disturbances and better recovery from errors [9]. Normally, human pilots initiate the flare by gradually pulling back the control column (or stick) to increase the elevator deflection [10]. After flare initiation, the already small engine thrust is gradually reduced to an idle throttle setting at touchdown [9].

A manually executed flare is therefore by nature a multivariable control task that requires precise coupling between timing (initiation) and action (force on the control(s)) $[10,11]$. The coupling between timing and action increases the task difficulty, but it has as benefit the provided trained operators with a compensation option. A late (or early) timing can be compensated by the application of a larger (or smaller) force on the control column [10].

Examining the pilot's actions is relatively easy, but retrieving the reasons behind these actions is much more difficult. How does the pilot know when to initiate the flare? What information is used to control the flare after initiation? These questions are discussed in the following paragraphs.

\section{Flare Initiation Strategies}

Even though the flare may be initiated in a different fashion when predictive guidance symbology is added to 
augment the natural cues, it is relevant to summarize the main findings from literature.

The flare initiation is based on at least two different timing strategies, the altitude perception strategy and the Time-To-Contact (TTC) strategy [10]. The disadvantage of the altitude perception strategy is that it is susceptible to ambiguity, especially if only a few visual cues are available. The TTC is defined as the time remaining to collision if no pilot control action is taken, and a strategy based on this variable offers a more robust flare timing strategy [10]. In theory, it can be registered by relating the distance to the runway aiming point to its time derivative, or by relating the visual angle (as defined from the pilot's eye(s) to both runway edges) to its time derivative. It was also found that more texture improves the perception of TTC and therefore pilot landing performance.

Evidence suggests that the TTC, or optical variable tau that describes the relative velocity of an optical image expanding across the retina, can be registered directly by the human eye [10-12]. Normally, pilots obtain many (visual) cues to adopt a correct timing and control strategy. However, during low visibility conditions the number of visual cues decreases and maintaining performance using manual control becomes more difficult or even impossible [13].

\section{Flare Control Strategies}

Then, what information is used for control during the flare? After initiation, the pilot has to apply the correct control action to reduce the descent velocity to an acceptable level, while assuring that touchdown is achieved at the desired runway spot. The correct control action varies with the initiation of the flare. A late initiation, for example, can be compensated by a larger control action and vice versa. The correct control action is also influenced by: the aircraft responses, the aerodynamic ground effect, and (aerodynamic) disturbances.

Unfortunately it is not known how the pilot determines the appropriate control action, nor the information that is used for feedback. Some may even argue if feedback is used at all, and suppose that the pilot executes a series of 'programmed' operations. Still, this is contradicted by evidence that suggests that the flare is controlled using the optical variable tau or TTC of one or multiple 'visual gaps' [14]. In the flare maneuver, the 'gaps' between the current and desired vertical speed and height need to be closed at the same time.

\section{SYNTHETIC VISION AND PREDICTIVE GUIDANCE}

From the above it is clear that executing a manual landing relies heavily on the availability of visual information from the out-of-the-window visual array. In low visibility conditions, these cues are limited or not available, and manual landings become impossible. Synthetic vision systems, which artificially restore the 'visual contact' with the ground, could resolve this issue. Providing synthetic vision with a three-dimensional perspective flight-path presentation, or 'tunnel-in-the-sky' has been shown to enable pilots to manually control the aircraft with very high precision [15] and to fly complex curved approaches and missed approaches that cannot necessarily be flown with current instrumentation $[16,17]$. To further improve performance, predictive guidance symbology can be added to the perspective flight-path display $[2-4,18,19]$.

Research on synthetic vision and perspective flight-path displays mainly focused on trajectory-following. The execution of the manual flare has rarely been studied. As discussed above, it is a transition between two straight trajectories, the glide path and the runway, and achieving a smooth landing is a difficult task. Although presenting the future aircraft's position has been shown to allow for a smoother anticipation of trajectory changes $[13,18,19]$, it is unclear whether this also holds for the final phase of the landing. Two predictive guidance concepts will be discussed in the following, developed in particular to support pilots in the final phase of the landing.

\section{The Flight Path Predictor}

The first predictive guidance concept that will be examined is the Flight-Path Predictor (FPP) [1-4]. The FPP presents the future aircraft's position a certain prediction time $T_{P R}$ ahead. Usually, the future position is presented by a cross shaped symbol, whereas a reference rectangle is shown ahead in the perspective display, essentially indicating where the predictor symbol is flying relative to the reference trajectory, commonly presented as a 'tunnel-in-the-sky'. When assuming that the prediction is accurate, the pilot's task when following the reference trajectory becomes a twoaxis pursuit tracking task with preview [13].

The flight-path predictor should now be confused with the Flight-Path Vector (FPV) symbol, commonly used on the Primary Flight Display. The FPV just shows the instantaneous direction of the aircraft's trajectory relative to the ground, the FPP shows the future position of the aircraft.

Basically, pilots will control the predictor symbol to move inside the reference frame marking where the predictor should be in the tunnel. When successfully keeping the predictor symbol inside the reference frame, the pilot controls the aircraft very precisely through the tunnel, as the aircraft will simply be at the position of the predictor symbol after the prediction time $T_{P R}$ has passed.

The FPP allows a smooth anticipation of changes in the trajectory to be followed $[1-4,13]$. When using predictive guidance, the transition from one path to the other, already starts at the moment the predictor symbol deviates from its reference trajectory. Hence, in the application studied here, the flare maneuver, the FPP could be vital in initiating and controlling the flare. Theoretically, at the moment the FPP 'hits' the runway, the pilot should start the flare. Clearly then, the chosen prediction time $T_{P R}$ is crucial to the flare initiation. When it is too large, pilots will start to initiate the flare too early, and vice versa.

One should keep in mind, however, that the prediction time also influences the tracking performance before the flare [20], so perhaps a compromise has to be found here between supporting the pilot during trajectory-following on the one hand, and timing the flare maneuver on the other 
hand. Apart from the prediction time, the accuracy of the position prediction also depends on assumptions of unknown future inputs. Hence, determining the right type of predictor laws and the 'best' prediction time is crucial to the success of using a FPP during the final phase of the landing. The optimal tuning of the FPP will be discussed in the following section.

\section{The Flight Trajectory Predictor}

The second predictive guidance concept that will be examined in this paper is the Flight Trajectory Predictor (FTP). This concept presents the whole predicted future trajectory, until the prediction time $T_{P R \max }$. It is possible to obtain the predicted trajectory using the same prediction techniques as the flight-path predictor, now for a range of prediction times $\left[0, T_{P R \max }\right]$. By 'connecting' the range of position predictions together and projecting them on each of the lateral tunnel sides, the trajectory of the future flight-path of the aircraft is directly visualized.

In this project, the predicted future position at the maximum prediction time $T_{\text {PRmax }}$ was shown using a circular symbol, visualizing the lateral path deviations in a way similar to the FPP. It is assumed that pilots use the full trajectory prediction and combines the furthest visible prediction of position and flight-path angle to control the aircraft during the flare. The intersection between the predicted path and the runway indicates the point where touchdown will occur if no pilot control action is taken, and the difference between the predicted flight-path angle and runway the inclination indicates the predicted descent speed at touchdown. These two variables are assumed to be the main factors that determine the success of the landing maneuver and pilots may be able to perceive and control both directly with this predictive guidance concept.

\section{OPTIMIZING THE PREDICTIVE GUIDANCE CONCEPTS}

In this paper the so-called 'extended predictor model', will be taken as the basis for developing the guidance laws for the flight-path predictor FPP and flight-trajectory predictor $[3,4]$. This predictor model, when tuned properly, is shown to require minimum pilot compensatory effort, is relatively insensitive to turbulence, and provides a high degree of face validity, as shown by theoretic findings and pilot-in-the-loop simulation experiments [4].

Through controlling the predicted future position, the predictor-aircraft system minimizes the actual path deviation after the prediction time has passed. The main goal when optimizing this pursuit tracking task is to minimize the compensatory effort that needed while maximizing performance [3, 4]. This introduces requirements for the dynamics of the controlled system that can be specified using manual control theory [21]. Accordingly, the predictor-aircraft $Y_{P R} Y_{a c}$ system should approximate a pure integrator $K / s$ with sufficient phase margin around the crossover frequency $\omega_{c}[21]$.

Other essential requirements to the pilot-predictoraircraft system $Y_{p} Y_{P R} Y_{a c}$ are system stability and low turbulence sensitivity. The extended predictor concept is capable of satisfying these requirements and can be expressed as [4]:

$$
\Delta h_{P R}(s)=\frac{K_{q}}{1+T_{q} s} q(s)+\frac{V T_{P R} s+V}{s^{2}} \dot{\gamma}(s)
$$

Here, $\Delta h_{P R}$ represents the predicted height error, $K_{q}$ is the pitch rate $q$ deviation feedback gain, $T_{q}$ is the lag time constant of this feedback loop, $V$ represents speed, $T_{P R}$ represents the prediction time, $s$ is the Laplace operator, and $\dot{\gamma}$ is the first time derivative of the flight path angle $\gamma$. A block diagram of the use of the extended predictor is given in Fig. (1).

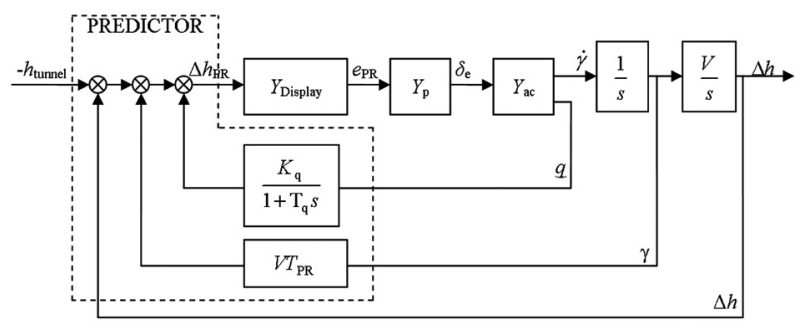

Fig. (1). Extended predictor diagram (after [4]).

To examine whether the predictor-aircraft system $Y_{P R} Y_{a c}$ approximates a pure integrator $K / s$ and satisfies the consistent requirement, the aircraft dynamics in the frequency region of concern should be determined. It is assumed that the Short Period (SP) mode of the aircraft dynamics is dominant in the frequency region of concern and where the pilot-predictor-aircraft crossover can be expected [4]. By selecting the lag time constant $T_{q}$ to be equal to the negative reciprocal of the stability derivative $Z_{\alpha}\left(T_{q}=-\frac{1}{Z_{\alpha}}\right)$ and selecting the pitch rate feedback gain $K_{q}$ to be equal to $V T_{P R} / \omega_{S P}$, it can be assured that the system to be controlled by the pilot approximates a pure integrator $K / s$.

Still, the prediction time $T_{P R}$ remains to be tuned to optimize the predictive landing guidance concepts for the final phase of the landing. A MATLAB/Simulink simulation of the pilot-predictor-aircraft system was used to investigate the system in the time domain for different values of prediction time to acquire the most favorable setting. The aircraft model $Y_{a c}$ used in this simulation represents a linearized Boeing 747, only the symmetrical motion is simulated. The full non-linear model of the Boeing 747 will be used in the pilot-in-the-loop simulator experiment discussed below.

The model is linearized around a stationary descending flight along the flight-path and it is assumed that this is valid during the final approach and flare maneuver until touchdown. The only control input that is used during this simulation is the elevator deflection. Adding the throttle as second control input would have offered better performance in the presence of disturbances [9], but the goal of this simulation is to optimize the predictive guidance concepts for the flare maneuver and not for the recovery from disturbances. 
The pilot model $Y_{p}$ that is used for this simulation consists of a pilot gain $K_{p}$ (the system to be controlled already approximates a pure integrator, so no equalization is necessary) and a model that represents the pilot's intrinsic limitations. In the following equation (2) the pilot model is given:

$Y_{\mathrm{p}}=\frac{\delta_{\mathrm{e}}(s)}{e_{\mathrm{PR}}(s)}=K_{\mathrm{p}} e^{-j \omega T_{\mathrm{e}}} \frac{1}{T_{\mathrm{N}} s+1}=K_{\mathrm{p}} e^{-03 j \omega \omega} \frac{1}{0.1 s+1}$

The symbol $\delta_{e}$ in equation (2) represents elevator deflection, $e_{P R}$ is the normalized predicted error in relation to the reference trajectory, $T_{e}$ and $T_{n}$ are respectively the effective (information processing) time delay and the neuromuscular lag [20]. The value of 0.3 seconds for the effective time delay is common for these types of control tasks, with integrator-like dynamics [21]. The only remaining variable in the pilot model that can deliberately be altered in the simulation is the pilot gain $K_{p}$, which effectively determines the balance between the stability and performance of the closed-loop pilot-vehicle system.

Stability is generally considered to be the most stringent requirement for system control. Using root locus design, a stable pilot gain $K_{p}$ region can be determined for different values of the prediction time $T_{P R}$ in the pilot-predictoraircraft system $Y_{p} Y_{P R} Y_{a c}$. The result is a stable pilot gain region with a maximum gain of approximately 2 for prediction times larger than two seconds and this stays roughly constant for larger prediction times.

After taking care of the stability requirement, the performance comes into play. To establish the optimal prediction time $T_{P R o p t}$, certain performance requirements must be determined. It is assumed that the two most important subjective measures that determine the landing performance are the runway consumption until touchdown $d_{r w}$ and the descent velocity at touchdown $\dot{h}_{t d}$. Therefore, it is stated that the landing maneuver is successful when these two measures are within the following limits [22]:

$d_{t d} \quad<366 \mathrm{~m}$
$\dot{h}_{t d} \quad<\quad 0.914 \mathrm{~m} / \mathrm{s}$

Using these limits, the following optimization procedure is defined [3]:

1) Run multiple simulations for different stable pilot gains and prediction times;

2) Evaluate if the performance of the simulated landing fulfils the requirements specified in equation (3) and can be labeled as successful; and

3) Count the number of successful landings over the whole range of stable pilot gains and prediction times to obtain the "landing score".

In Fig. (2) the results of the optimization procedure, are effectively captured in the "landing score", it is illustrated. It can be observed that for both the predictive guidance concepts (FPP and FTP) the optimal (maximum) prediction time is located at approximately five seconds. Hence, in the following a prediction time of 5 seconds is used for both concepts.

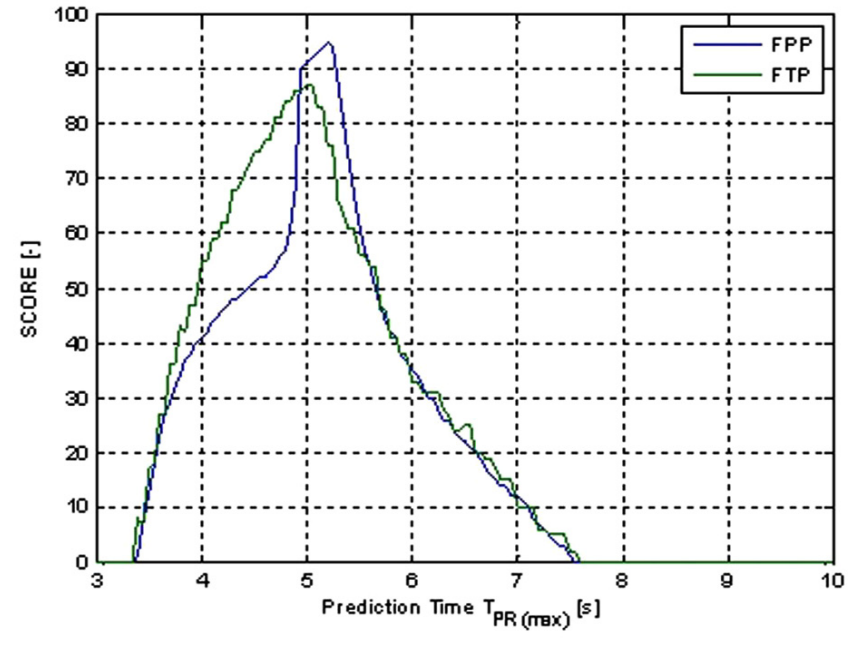

Fig. (2). Landing score, the summation of successful simulated landings versus prediction time $T_{P R}$.

Now the system has been optimized using manual control theory and offline simulations, the human interaction with this control system needs to be examined to complete the investigation.

\section{EXPERIMENT}

The goal of the experiment was to evaluate the usefulness of the two predictive landing guidance concepts discussed in the previous sections, in combination with a synthetic vision display. Apart from trajectory-following performance, our main interest lies in pilot performance in initiating and controlling the landing flare.

\section{METHOD}

\section{Apparatus}

The experiment was conducted in the NLR Generic Research Aircraft Cockpit Environment (GRACE) reconfigurable moving base flight simulator. For this experiment the GRACE simulator was configured to simulate a non-linear Boeing 747-400 aircraft for both hardware (steering column and Boeing throttles) and software components.

\section{Subjects and Instructions to Subjects}

Ten professional airline pilots participated in the experiment. The mean age of the pilots was 39 years and they had an average flight experience of 7,237 hours (Table 1). All pilots were instructed to fly the final phase of the landing as they would normally do during a manual landing, with the exception that they could manually land the aircraft using instruments only. That is, their usual guidance for manual approach-to-landing tasks, the flight-director, was not available.

Two-pilot crews were formed, in which the pilot-incontrol was considered the test subject, and the other pilot the observation pilot. After the experimental conditions were completed for the pilot in control, both pilots changed their roles and the experiment proceeded with the other pilot acting as pilot in control. To create a more natural 
Table 1. Characteristics of the Pilot Subjects in the Experiment

\begin{tabular}{|c|c|c|c|c|}
\hline Pilot & Gender & Age & Hours & Types of Aircraft \\
\hline \hline A & M & 42 & 11,700 & B747-300, B767-300 \\
\hline B & M & 34 & 6,015 & B737-200/300/400/500/700, A330, A320 \\
\hline C & M & 42 & 8,900 & NF-5, F-16, B747-300, B737-300/900 \\
\hline D & M & 33 & 6,700 & Fokker 100, B767, B747-300, B777, B737 \\
\hline E & M & 53 & 13,000 & DC-9, DC-10, B737-400, B747-400 \\
\hline F & M & 36 & 3,000 & BAe 146/Avro RJ \\
\hline G & M & 40 & 5,100 & Cessna Citation II, Fairchild Metro II, Fokker 100, B767, A330 \\
\hline H & M & 24 & 1,150 & Cessna 172/206/208/182, Piper Pa28, B777 \\
\hline I & M & 56 & 13,000 & F5, F104, DC-10 Fokker F27, A310, B747-300, B747-400 \\
\hline J & M & 30 & 3,800 & MD-80, MD-11, B737 \\
\hline
\end{tabular}

environment, pilots were allowed to communicate with each other during the experiment on any matter. When completing the questionnaires, discussed below, however, the pilot in control was in charge of answering the various queries, and also filled in the workload sheet all by himself.

\section{Independent Variables}

Only one independent variable was varied in the experiment, namely the type of visual information provided to the pilot. Five different flight displays were used, illustrated in Figs. (3-7), and referred to as D1 to D5 in the following:

D1 A conventional PFD, used in conjunction with a degraded but still available outside-vision presentation in the simulator;

D2 A synthetic vision display with Flight-Path Predictor (FPP), without outside vision;

D3 A synthetic vision display with Flight Trajectory Predictor (FTP), without outside vision;

D4 A synthetic vision display with Flight-Path Vector (FPV), without outside vision; and

D5 A synthetic vision display with Flight-Path Vector (FPV) with better textures available in the synthetic visual display, without outside visibility.

In all synthetic vision displays the future trajectory was shown as a 'tunnel-in-the-sky', the width of the tunnel being constant at $50 \mathrm{~m}$. The display used had a size of 14,1 inches and was positioned right in front of the pilots, at a distance of approximately $90 \mathrm{~cm}$.

The rationale of adding condition D5 to the experiment was to investigate the possible improvement of a pilot's landing performance when more and better texture was available from the synthetic display.

\section{Experimental Design and Procedure}

The five experiment conditions were organized in a quasi-random fashion, taking a good balancing of conditions over subjects in account. Conditions were spread over the experimental days in blocks of five runs. On each day, a crew of two pilots was invited to participate in the experiment. After conducting several training runs for each experimental condition they were asked to complete five replications of all conditions that served as measurements. When the runs for the pilot-in-control were done, the pilots changed their positions and the other pilot became the pilotin-control, and the experiment was repeated.

Each run started at a height of 1,000 feet and it took about 90 seconds for pilots to complete the landing. After each landing, the pilot in control was asked to complete an 'end of run questionnaire' which examines the workload using the NASA TLX Subjective Workload Scale [23]. Subjective landing performance was determined by asking the pilots to rate the main performance parameters, touchdown distance from threshold and sink rate, after touchdown.

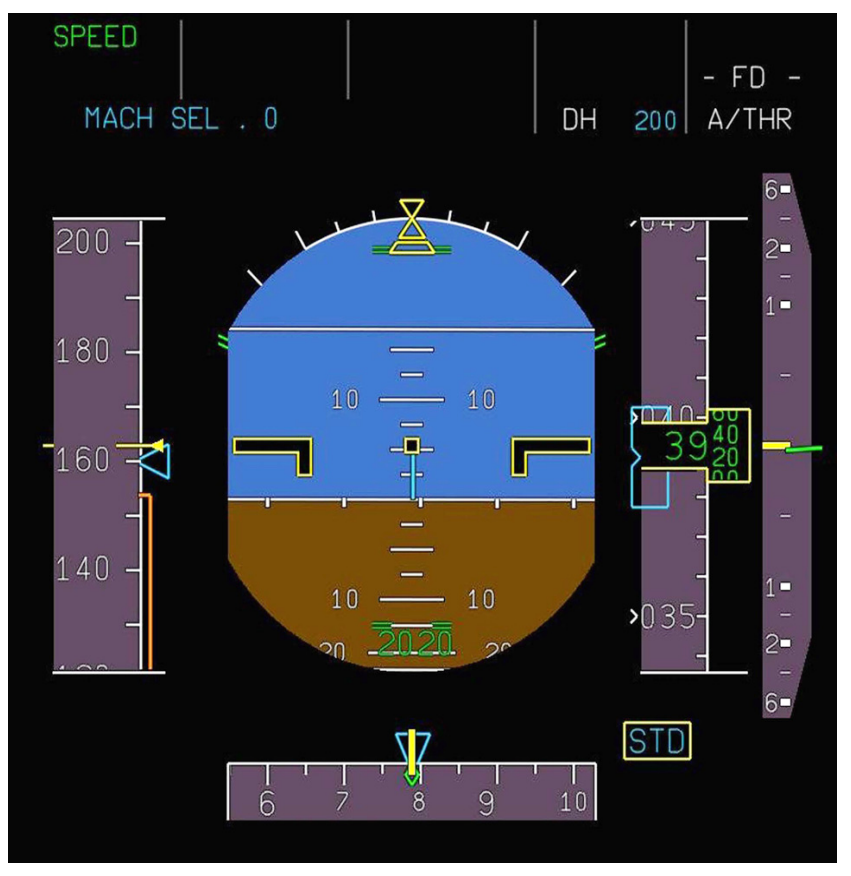

Fig. (3). Conventional PFD (D1). 


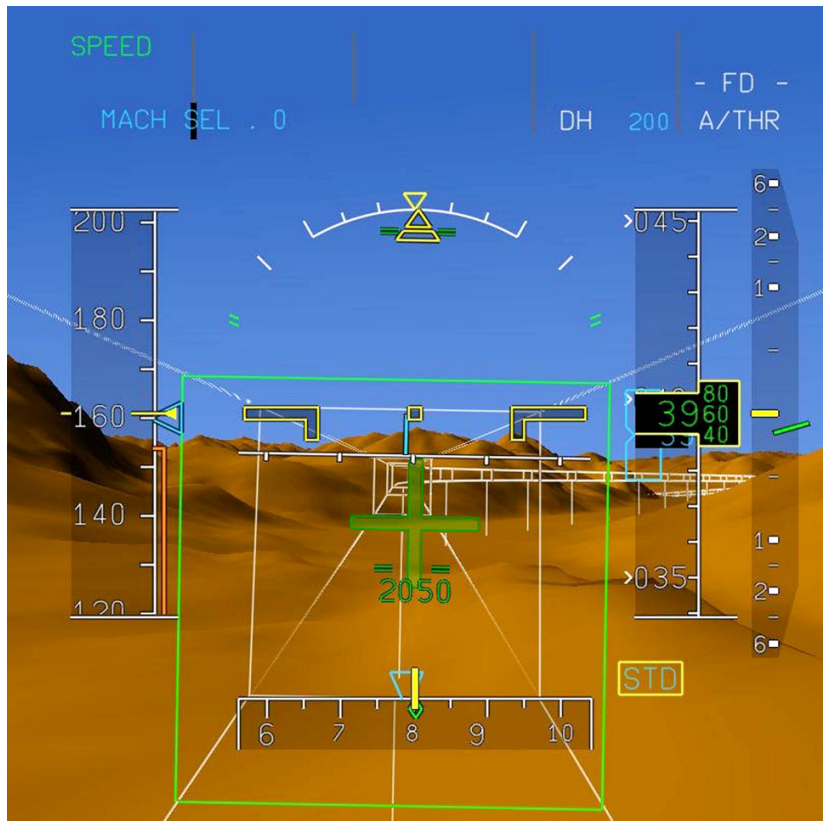

Fig. (4). Synthetic vision display with FPP (D2).

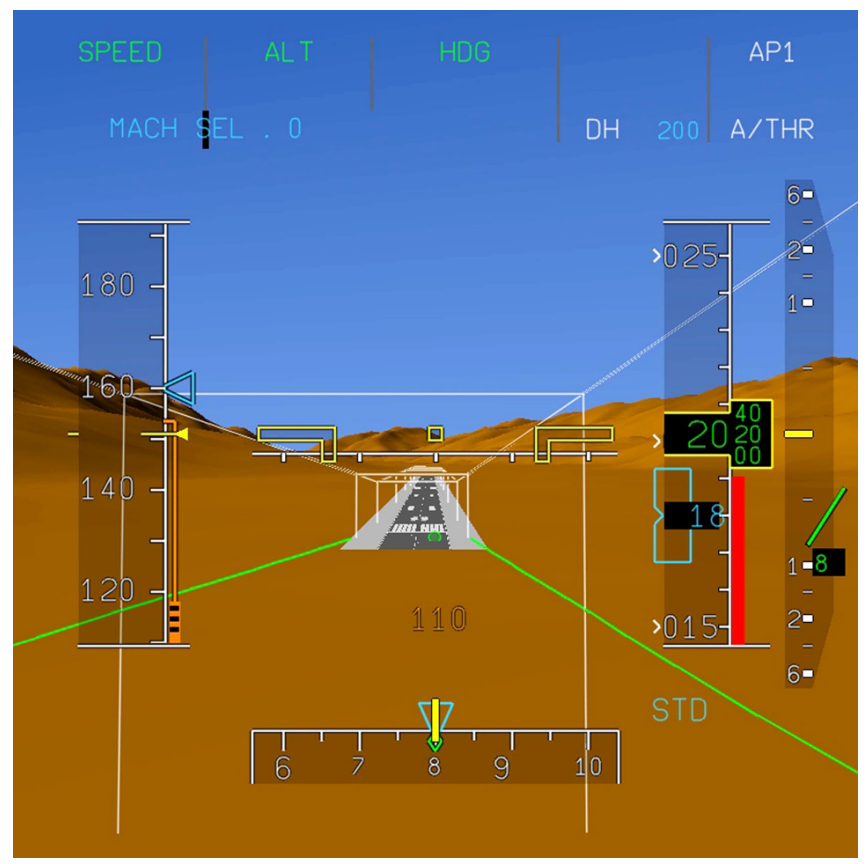

Fig. (5). Synthetic vision display with FTP (D3)

After all runs for a particular condition were completed, the pilots were asked to fill in an 'end of block questionnaire' that was used to obtain comments about that specific experiment condition. At the end of the day when all experiment cases were conducted, pilots were asked to complete the 'end of day questionnaire' to explain their strategies, rate each condition and comment on the experiment.

\section{Dependent Measures}

Six types of variables acted as dependent measures: 1) pilot control activity, expressed in the standard deviation (STD) of aileron deflection $\delta_{a}$, and elevator deflection $\delta_{e} ; 2$ ) pilot workload, expressed in the TLX workload ratings which were converted into z-scores; 3) path-following performance, expressed in the STD of the horizontal and vertical track position error $x_{e}$, and $y_{e}$, respectively; 4) landing performance variables, that is, the mean runway consumption until touchdown, and the mean sink rate at touchdown; 5) flare initiation variables, that is the mean flare initiation height and the mean Time-To-Contact; 6) flare control variables after initiation, that is, the mean pitch angle at touchdown $\theta_{t d}$, the mean of the maximum elevator excitation, the mean time to maximum elevator excitation, and the mean flare excitation rate.

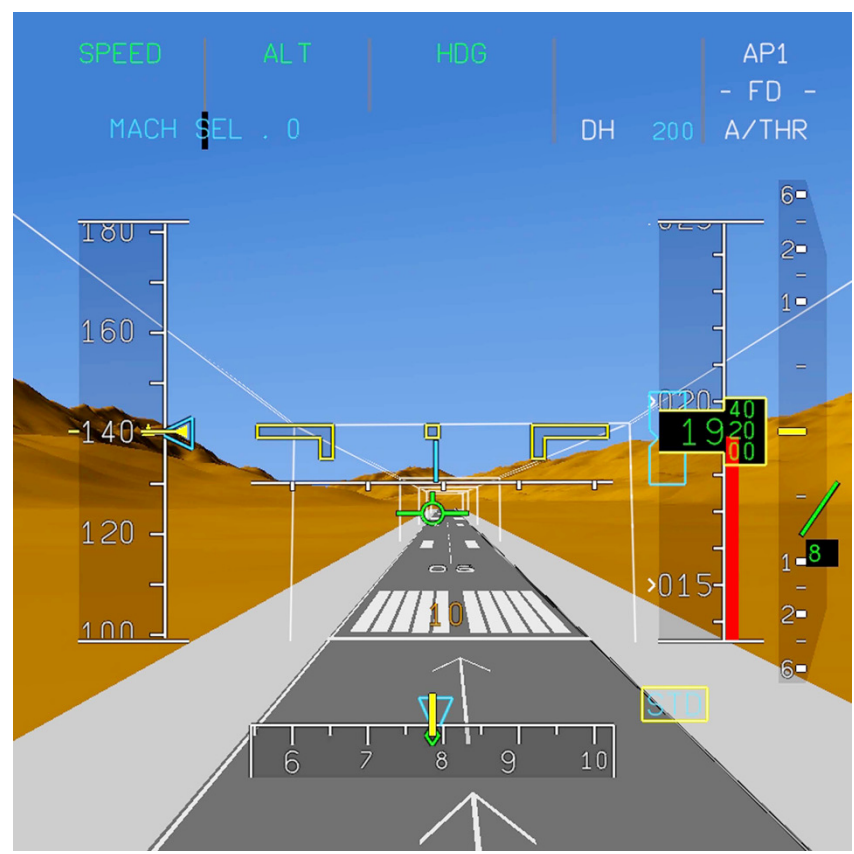

Fig. (6). Synthetic vision display with FPV (D4).

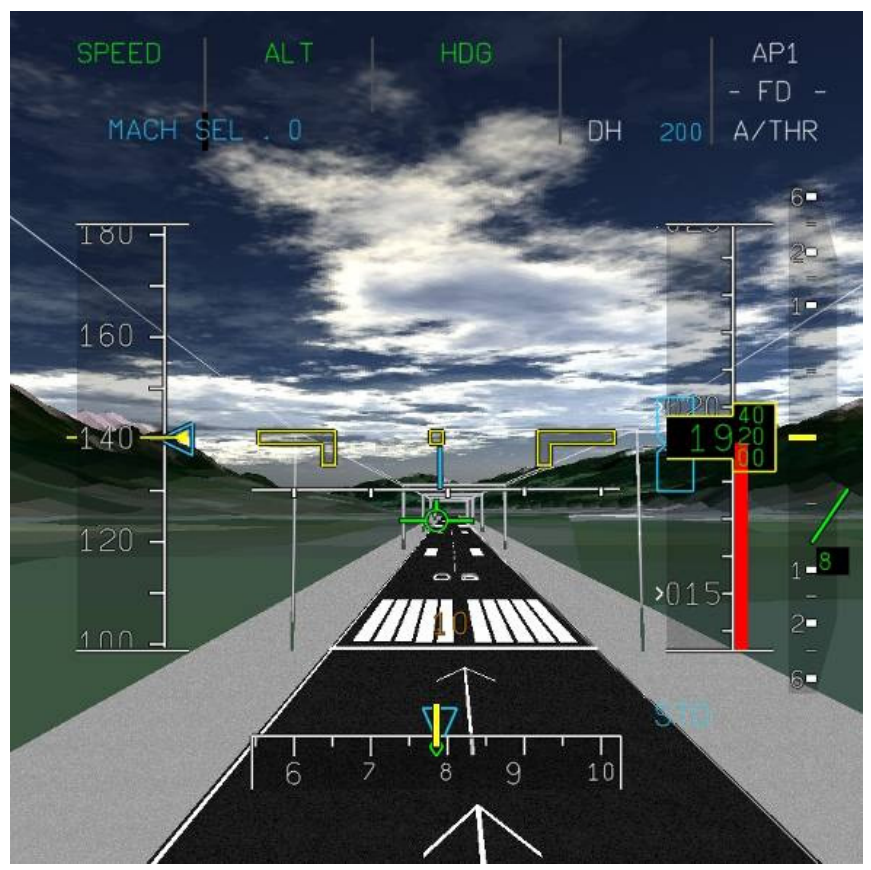

Fig. (7). Synthetic vision display with textures and FPV (D5).

Apart from the flare-related properties, which were estimated based on an algorithm discussed in the Appendix, 
all other variables were measured in the interval between 1,000 feet and touchdown. In the experimental data analysis, an analysis of variance technique (ANOVA) was used, if applicable followed by Student Newman-Keuls post-hoc analyses.

\section{EXPERIMENT HYPOTHESES}

It was hypothesized that the addition of predictive guidance to the synthetic vision displays would result in lower pilot control activity, lower workload, and better (path-following) performance as compared to synthetic vision displays without augmentation (D4, D5). For the difference between the FPP (D3) and the FTP (D4), it is hypothesized that the FPP has better path-following performance, while the FTP has better landing performance. The addition of textures to the synthetic vision display (D5) was hypothesized to improve landing performance. The conventional display with available outside visibility (D1) is expected to result in the best landing performance of all displays, as here the visual array mimics the natural cues the best.

\section{RESULTS AND DISCUSSION}

The main results of the experiment are summarized in this section.

\section{STATISTICAL ANALYSIS OF THE DEPENDENT MEASURES}

The means and $95 \%$ confidence limits of the dependent measures are shown in Figs. (8-19).

\section{Pilot Control Activity}

For elevator control inputs (Fig. 8), the control activity tends to be larger for conditions D2 (FPP) and D3 (FTP) $\left(F_{4,36}=0.483\right.$; not significant). Clearly, the predictive guidance concepts cause the pilot to control the aircraft symmetric motion more actively as compared to the other experiment conditions.

Student Newman-Keuls (NK) post-hoc analysis ( $p=$ 0.05 ) indicates that the aileron control activity (Fig. 9) is largest for the condition D4, the synthetic display with FPV but without textures. The difference between condition with and without textures (FPV $+\mathrm{T}$ and FPV respectively) is interesting, because it indicates that the addition of textures to the synthetic vision display resulted in less control activity.

The results for the conditions with predictive guidance (FPP and FTP) indicate that the addition of predictive guidance supports pilots in lateral control and allows them to adapt a less active control strategy compared to the FPV condition that provided neither predictive guidance nor additional visual information using textures. Between these two conditions the control activity with FPP tends to be smallest, while both use the same predictor laws and related symbology. As discussed above, the FTP also provides a small circular symbol at the end of the predicted trajectory, and its saliency may be an important support for pilots.

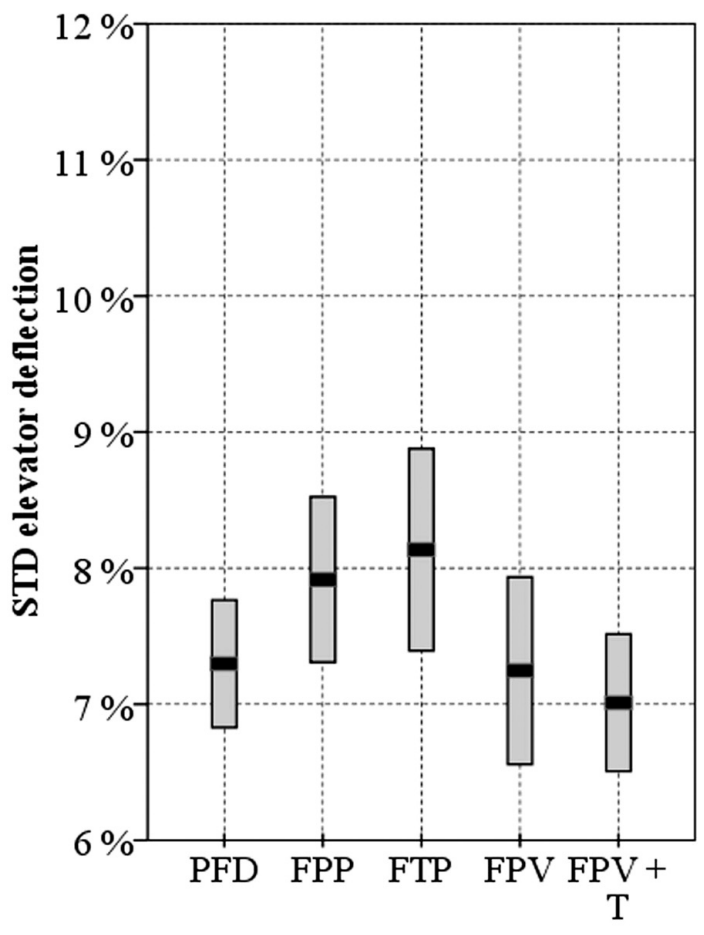

Fig. (8). STD elevator deflection $\delta_{e}$

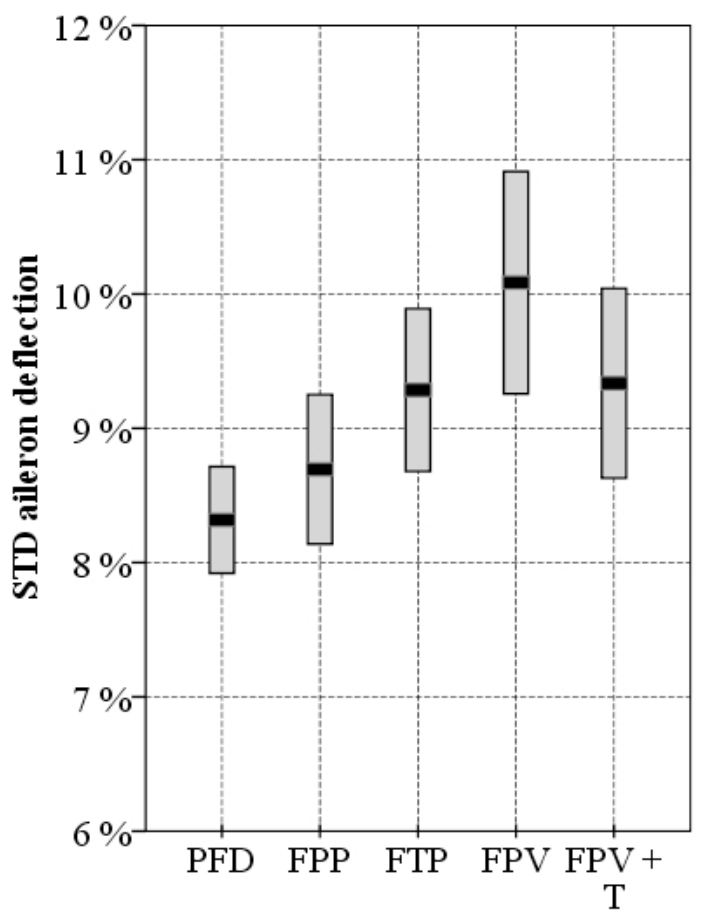

Fig. (9). STD aileron deflection $\delta_{a}$

\section{Pilot Workload}

The workload for condition D1, with conventional PFD and outside visibility, was rated lower than all other conditions (Fig. 10). With the exception of condition D3 (the FTP), the differences in workload for the synthetic vision conditions are small. The FTP is specifically designed to 
support the flare maneuver and does not provide much assistance before this moment. Pilots commented that a better option would be to enable the FTP just before the flare initiation to prevent the added symbology from becoming a nuisance. This nuisance is probably the main cause of the higher rated workload, the frustration level component of the TLX rating was generally rated higher for the FTP.

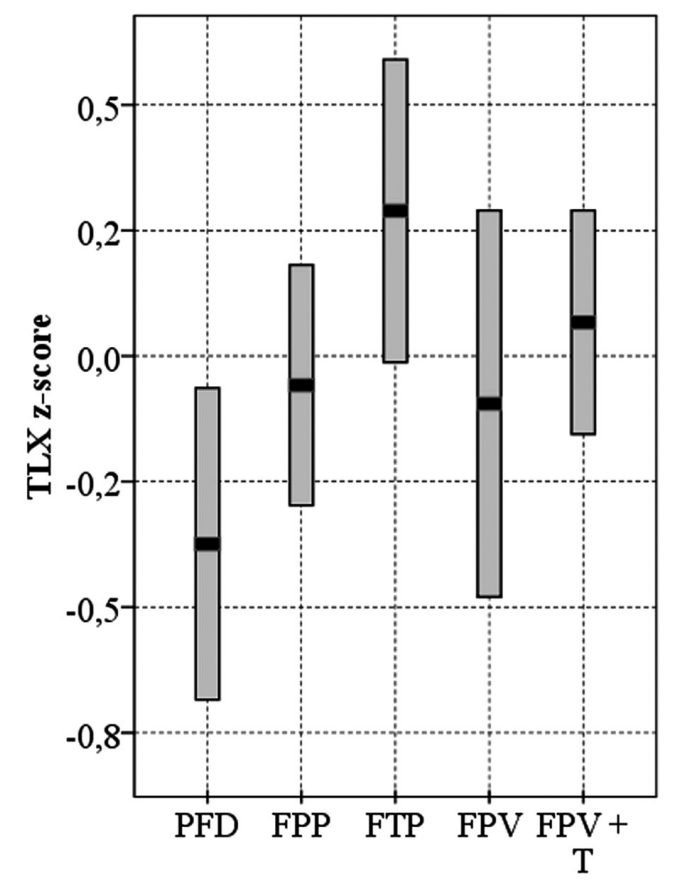

Fig. (10). TLX workload rating (z-score).

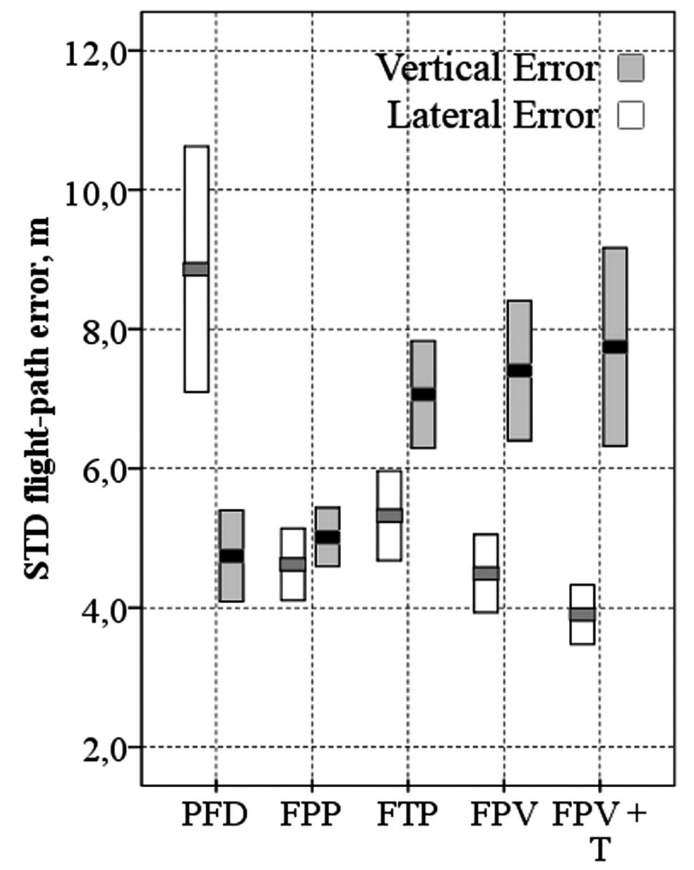

Fig. (11). STD lateral and vertical flight-path error $x_{e}$ and $y_{e}$.

\section{Path-Following Performance}

The pilot controls the aircraft from a height of $1,000 \mathrm{ft}$ to touchdown and the flare is generally initialized at a height of $50 \mathrm{ft}$. The pilot has to control the aircraft for $95 \%$ along the glide-path to approach the runway and, as the old saying goes: "A good landing requires a good approach", it is an important part of the landing. The path-following performance is illustrated in Fig. (11). Post-hoc analysis $(\mathrm{NK} ; p=0.05)$ revealed that the vertical path-following performance is significantly better for experiment condition with PFD and with FPP $\left(F_{4,36}=4.025 ; p<0.01\right)$.

Observing the elevator control activity for these experiment conditions from Fig. (8) and comparing this to the path-following performance indicates that the higher control activity for the experiment case with FPP did not result in an increased accuracy. Still, a synthetic vision display with FPP is clearly able to assure a vertical path following accuracy that can be compared with conducting a conventional landing with available outside visibility. For the experiment case with FTP, the control activity tends to be even larger, while the path-following performance decreases. As mentioned before, the FTP was designed to support the pilot during the flare maneuver and during the approach the pilot has to control the aircraft through the tunnel without much additional support. This has probably resulted in the lower path-following accuracy for this predictive guidance concept.

The lateral path-following performance is clearly less accurate for the D1 condition with PFD $\left(F_{4,36}=18.35 ; p<\right.$ 0.01 ). Still, the aileron control activity does not vary much between experiment conditions (especially not between experiment case with PFD and FPP) (Fig. 9). This difference may be caused by the relatively small sized tunnel that is used on the synthetic vision displays compared to the larger 'lateral tolerance' that is 'allowed' during conventional approaches. Pilots commented that the tunnel size for the synthetic vision concepts may be too small at high altitudes. This tunnel size was optimized for the flare maneuver and kept constant during the approach, but a larger tunnel that narrows with decreasing height may be more practical.

\section{Performance Variables}

As mentioned previously, it is assumed that two dependent measures are of main importance to the flare maneuver. These are the runway consumption until touchdown; and the vertical descent velocity at touchdown. Two requirements for these measures were introduced to determine if a landing is successful or not.

First, the runway consumption until touchdown is considered. In Fig. (13) the means and confidence limits indicate small differences between experiment conditions $\left(F_{4,36}\right.$ $=0.686$; not significant). The runway consumption for successful landings is required to be smaller than 366 meters. Fig. (13) shows that the runway consumption during the actual experimental runs lays around this requirement and the runs that are represented by the area below this requirement can be labeled as successful. When comparing the different 
experimental conditions, the general trend is that the runway consumption is smallest for condition D1 with PFD and outside visual, and increases for the subsequent conditions D2 until D5, resulting in fewer successful landings.

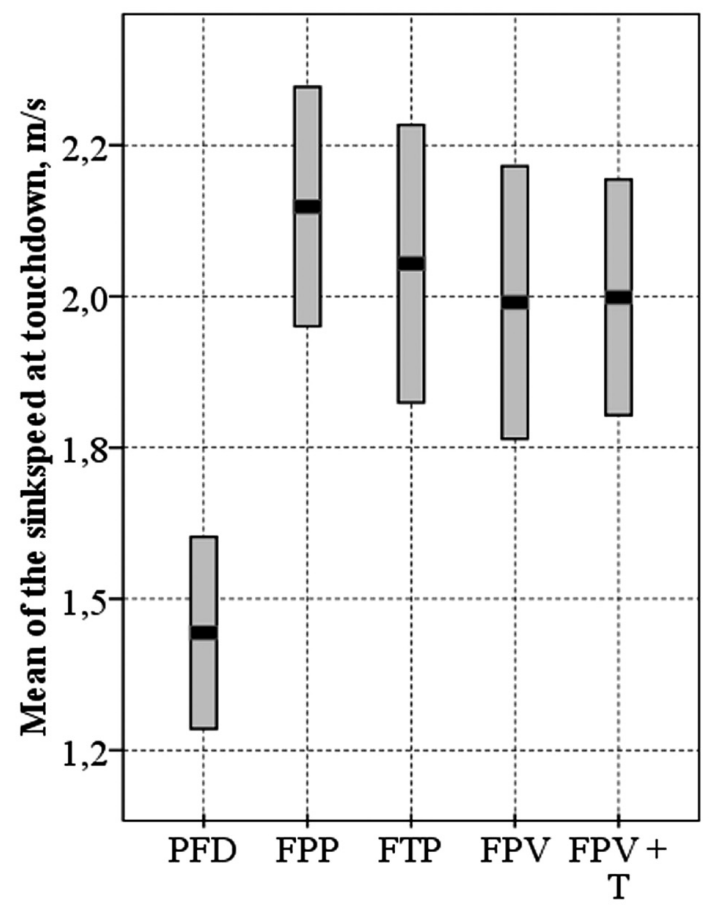

Fig. (12). Mean sink rate at touchdown.

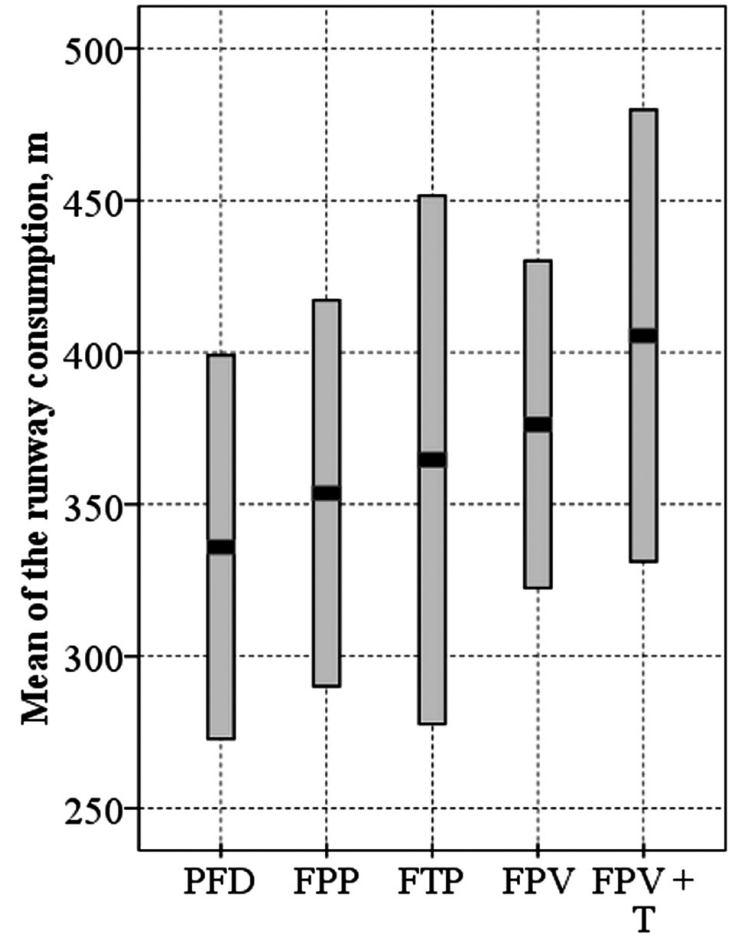

Fig. (13). Mean runway consumption until touchdown.

Second, the sink rate at touchdown will be discussed. Fig. (12) shows that none of the confidence limits are below the maximal sink rate requirement of $0.914 \mathrm{~m} / \mathrm{s}$ for successful landings. Post-hoc analysis (NK; $p=0.05$ ) revealed that the sink rate for the conventional experiment condition with PFD with outside visual is significantly smaller as compared to all other conditions, as was hypothesized $\left(F_{4,36}=7.514 ; p<0.01\right)$. What may have caused the striking difference in sink speed between 'normal' landings with available outside vision and landings using synthetic vision as sole means of visual information? Is it caused by a wrong flare initiation or is the flare control after initiation more likely to be the cause? To answer this questions, the flare maneuver has been examined in more detail, discussed in the following paragraphs.

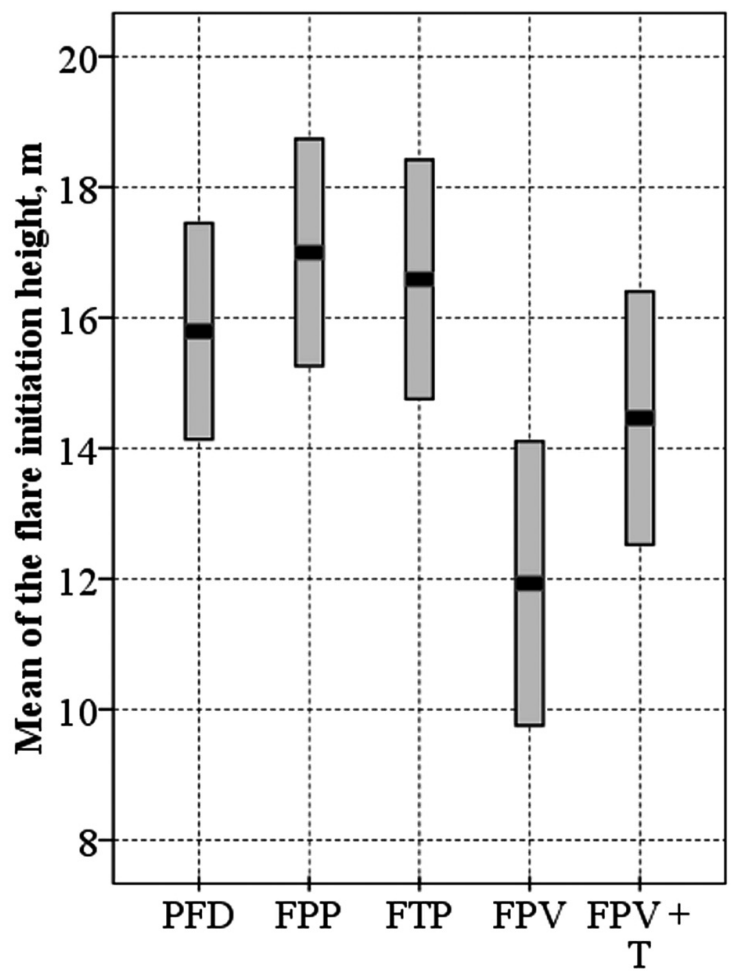

Fig. (14). Mean flare initiation height.

\section{Flare Initiation Variables}

The flare initiation time instance had to be determined in order to obtain an indication of whether it affected the observed differences in sink rate. The technique that was developed to determine the flare initiation moment is explained in more detail in the Appendix. In short, the technique uses the elevator angle to reveal the moment when the pilot pulls back the control column to excite the phugoid eigenmotion of the aircraft.

As mentioned previously, the pilot uses at least two different flare initiation timing strategies; one based on the perception of altitude and one on the notion of Time-ToContact (TTC). For this reason, the flare initiation time instances were converted to the flare initiation height and TTC. By illustrating the means and confidence limits (Figs. $14,15)$ for these two important variables, an indication of the likelihood that the flare initiation influences the sink rate at the end of the entire maneuver can be derived.

Starting with initiation height, the mean flare initiation height was located between 15 and $18 \mathrm{~m}$ (or 50-60 ft) on average (Fig. 14). This is noticeable for all experiment 
conditions with exception of the condition for synthetic vision displays with FPV but without textures. Post-hoc tests $(\mathrm{NK} ; p=0.05)$ showed that the flare initiation height for this experiment case is clearly smaller than the other experimental conditions. Notice that there is no distinct difference between the conventional experiment condition with PFD and the two experiment conditions with synthetic vision and predictive guidance (FPP and FTP).

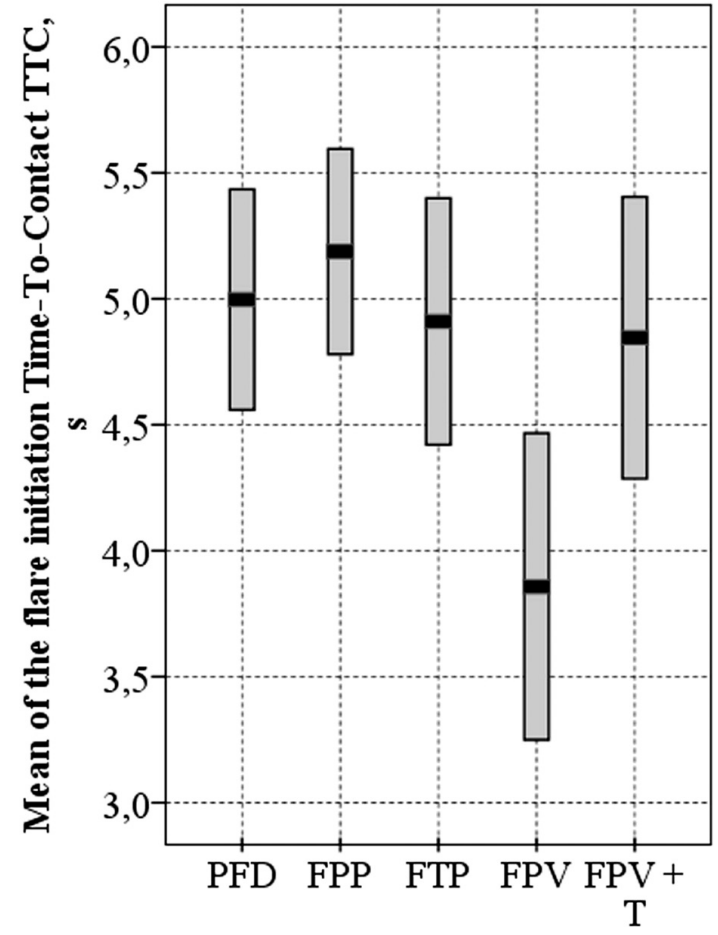

Fig. (15). Mean flare initiation Time-To-Contact.

Equivalent to the theoretically determined optimal time to initiate the flare maneuver, the observed flare initiation TTC is approximately 5 seconds (Fig. 15). Although there are no significant differences between the experiment conditions $\left(F_{4,36}=1.883\right.$; not significant $)$, the general trend can be compared with the trend in the flare initiation height (Fig. 14). Pilots tend to initialize the flare maneuver later and at a lower height when no predictive guidance or textures are displayed on the synthetic vision display (FPV condition without textures).

Consequently, there are no indications that the moment of flare initiation contributes to the difference in sink rate between the conventional condition, with PFD and outside visibility, and the other conditions that provide synthetic vision. The general trends of the moment of flare initiation do not seem to match to the trend in sink rate over the experimental conditions. In the following paragraph, the flare control after initiation will be examined to determine if there are indications that this aspect contributes to the differences in sink rate.

\section{Flare Control Variables After Initiation}

After initializing the flare, the pilot controls the aircraft until touchdown finalizes the maneuver. The pilot rotates the aircraft in pitch direction and the resulting pitch angle at touchdown is illustrated in Fig. (16). Post-hoc tests (NK, $p=$ 0.05 ) showed a clear difference between pitch angle at touchdown for the conventional experiment condition with PFD and the other conditions $\left(F_{4,36}=2.869 ; p<0.05\right)$.

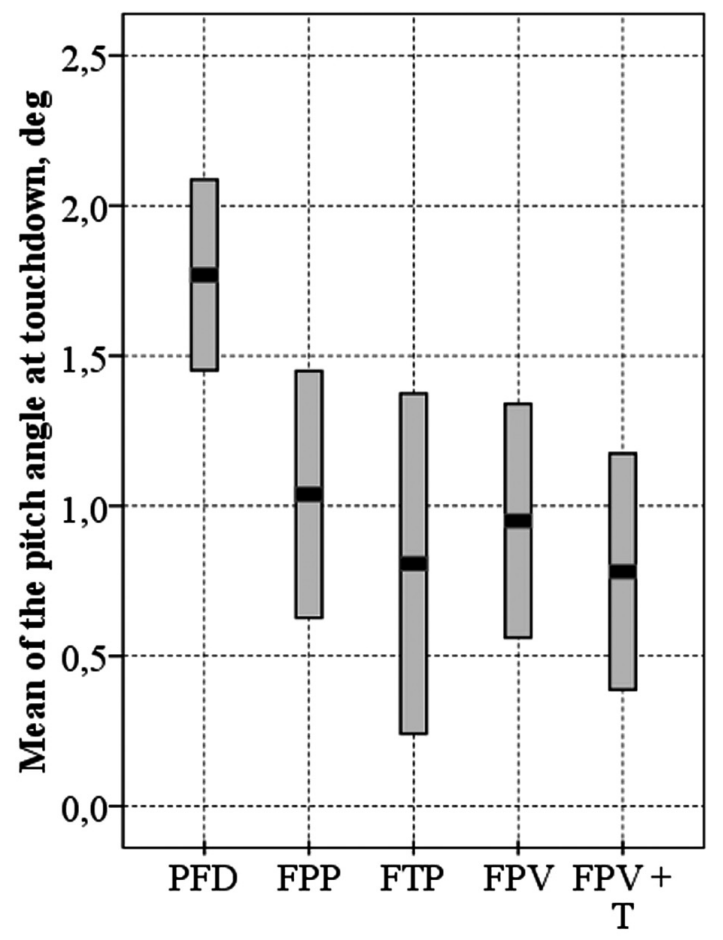

Fig. (16). Mean pitch angle $\theta_{\mathrm{td}}$ at touchdown.

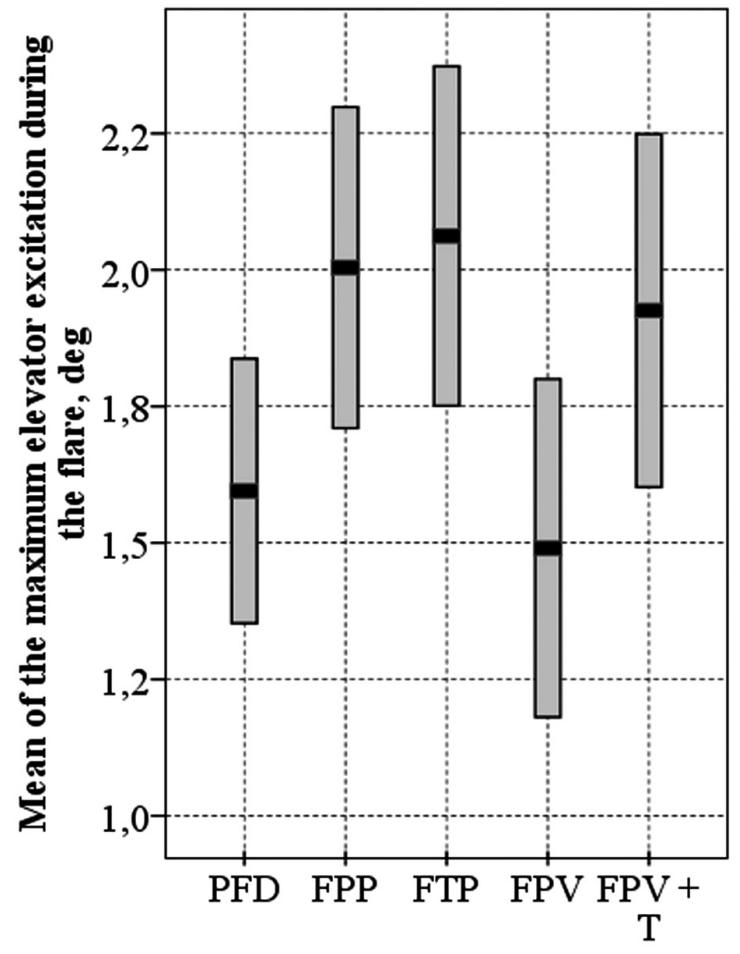

Fig. (17). Mean of the maximum elevator excitation during the flare.

To increase the understanding of how this pitch angle is achieved and how the flare is controlled during the experiment, the change in elevator deflection is examined. Pilots normally initialize the flare maneuver by gradually 
pulling back the control column to increase the elevator deflection. It is assumed that the maximum elevator deflection after initiation and the time between initiation and reaching this maximum deflection are of strong influence to the flare maneuver. To establish these two variables, the original elevator control signal is processed by a Moving Average Filter (MAF) with an interval length that equals the period of the short period eigenmotion (also see the Appendix). This processed signal is then used to determine the difference between maximum elevator deflection and the elevator deflection at flare initiation. Also the time difference between these two occurrences is determined. The means and confidence limits of both of these differences are given in Figs. $(17,18)$.

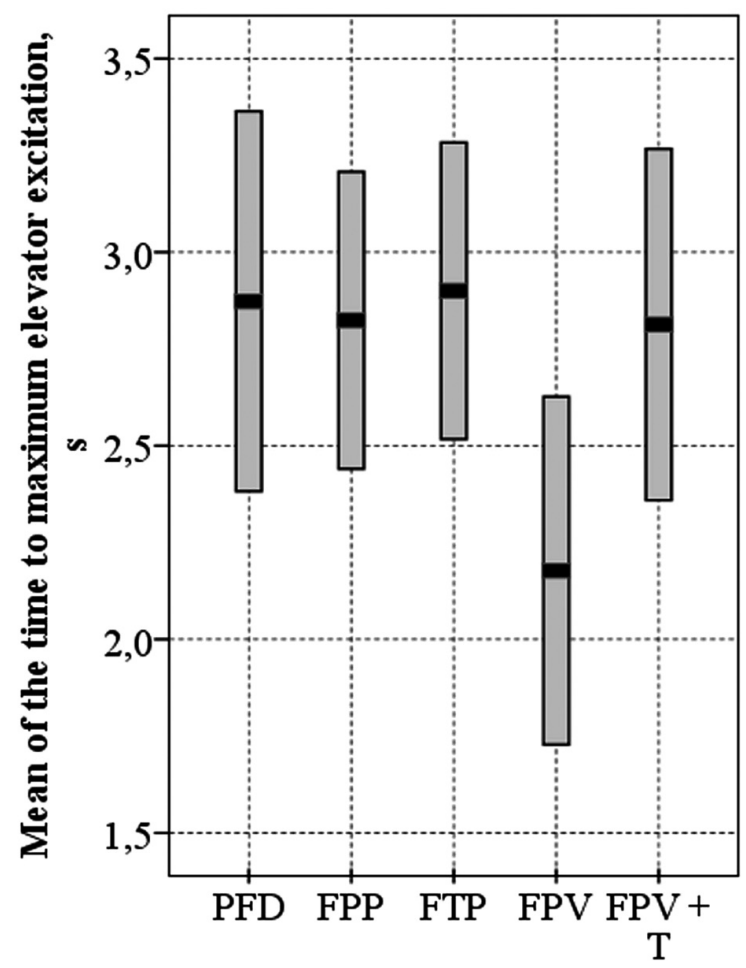

Fig. (18). Mean of the time to maximum elevator excitation during the flare.

The maximum elevator deflection tends to be smaller for the conventional experiment condition with PFD (D1) and the condition with FPV (D4) $\left(F_{4,36}=1.636\right.$; not significant $)$ (Fig. 17). For the time to maximum elevator deflection, however, only the experiment condition with FPV seems smallest $\left(F_{4,36}=1.129\right.$; not significant) (Fig. 18). Thus, for the condition with FPV both the maximum elevator deflection and time to reach this maximum tend to be relatively small. Combining these two variables by dividing the maximum deflection with the time to obtain this maximum from initiation will provide an elevator deflection rate, which may contribute to increasing the understanding of the flare control after initiation. The means and confidence limits of this rate are given in Fig. (19). This figure indicates that the elevator excitation rate tends to be smaller for the conventional experiment condition $\left(F_{4,36}=\right.$ 0.359; not significant). In other words, pilots tend to pull back the control column more gradually when outside vision is available (the conventional condition with PFD, D1), compared to landings with synthetic vision as a sole means of visual information.

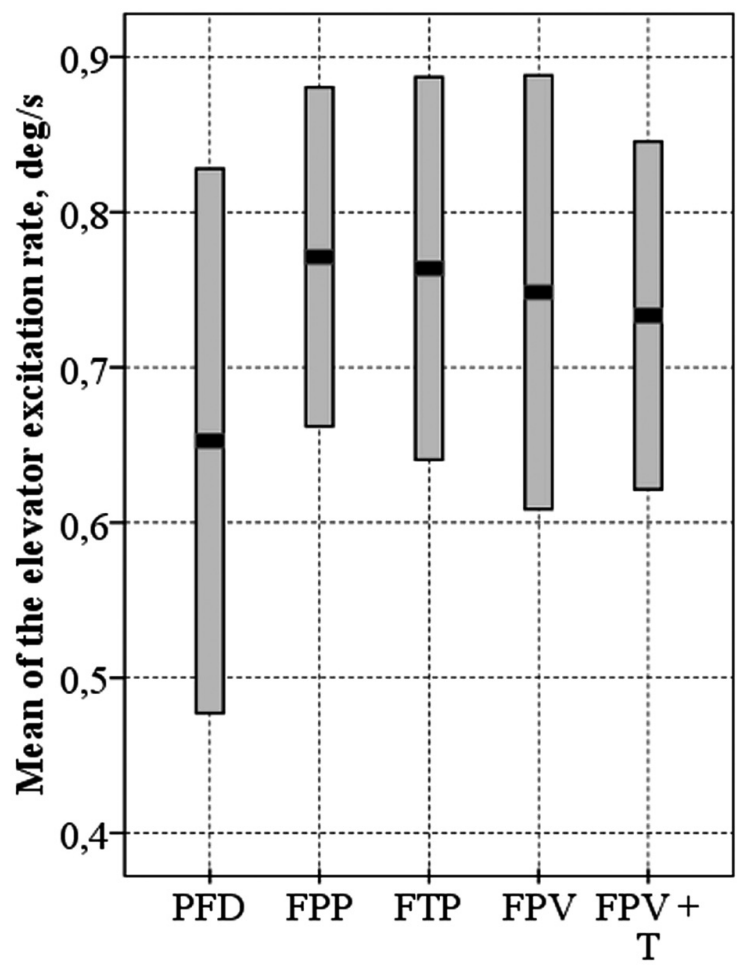

Fig. (19). Mean of the elevator excitation rate.

Notice the similarities between the elevator excitation rate (Fig. 19), the pitch angle (Fig. 17), and sink rate at touchdown (Fig. 12). Comparing these three figures provides indications that the flare control after initiation is indeed the cause of the differences in sink rate at touchdown for landings performed using outside vision and landings using a synthetic vision display only. It seems that pilots pull back the control column too abruptly when only synthetic vision is provided. A more gradual control strategy is likely to enhance the performance during the flare maneuver.

\section{PILOT QUESTIONNAIRE}

Almost all pilots suggested that the pitch ladder and banking angle indications should be included in the synthetic vision displays. These were left out to prevent clutter, but pilots suggested that this symbology is crucial during landing tasks.

Two pilots suggested that the size of the tunnel-in-thesky should be larger at greater heights to prevent pilots from controlling the aircraft with too much precision when it is not absolutely necessary. Two other pilots even suggested that the tunnel-in-the-sky in the synthetic vision displays should be removed, or replaced by a $2 \mathrm{D}$ indication of the flight path to be followed.

Three pilots suggested that the conditions with FPP and FTP symbology should only be displayed below an altitude of 200 feet. Also, one pilot indicated that a target symbol for the FPP and FTP within the tunnel should be included. According to one pilot, adding wind information (direction and intensity) to the PFD may be helpful during the final phase of the landing. One participant indicated the need for 
more training and would have liked to fly more experimental runs with the displays that include FPP and FTP symbology.

Regarding the experimental setup, it was mentioned that the experiment day was very intense. The experiment consisted of a large amount of manual performed landings. This large amount caused a few pilots to be rather tired at the end of the day.

For future research on this subject, all pilots were asked if they could think of any other way to support the manual execution of the flare maneuver. Three pilots suggested that presenting the synthetic environment and symbology on a Head-Up-Display (HUD) could be advantageous. Surprisingly, three pilots mentioned that they would prefer direct control commands, like those given by a Flight Director (FD) but in this case related to the flare maneuver.

\section{DISCUSSION}

In the experiment, only for a very few landings the performance requirements were satisfied. Although the consumed runway distance generally remained within limits (Fig. 13), overall the landings were too hard during this experiment (see Fig. 12). Therefore, it is not possible to assess the number of successful landings and compare these for the different experimental conditions as was done in the optimization procedure.

The performance (especially the sink rate) at touchdown of the conventional PFD display condition with outside visibility was significantly better (D1) than the conditions that only provided a synthetic vision display. Although this was hypothesized, the differences in pilot performance were unexpectedly large.

A possible reason for this large difference was the nonconformal field-of-view on the synthetic vision displays. In order to present more information on the synthetic vision display, the field-of-view was chosen to be relatively large $\left(60^{\circ}\right)$. The synthetic field-of-view did not match the pilot's eye field-of-view, which was less than $20^{\circ}$ when one was focusing on the 14.1 inch display at a distance of $90 \mathrm{~cm}$. The pilot may have (unconsciously) assumed that the synthetic vision display resembled the view when looking out of a window that is the same size as the display. The perceptual bias that results from this "window assumption" [24] may have caused objects to appear to be located in a different position than in actuality.

Another probable cause was the relatively small sized display with respect to the simulator outside vision display, affecting the perception of aircraft rates and accelerations crucial for manual control. These two causes are believed to have influenced the pilot control strategy and may explain the observed performance differences.

By analyzing the pilot's control actions it was noticed that the pilot control strategy after flare initiation was the main cause of the harder landings. Pilots pulled back the control column in a rather abrupt fashion when using the synthetic vision display. A more gradual control action, such as applied with the condition with the outside visual (D1) would have improved landing performance, but the synthetic vision display probably did not provide enough salient visual information to allow pilots to adopt such a more gradual control strategy.

Fig. (15) indicates that the flare maneuver was initialized at lower height and smaller Time-To-Contact for the experimental condition with FPV compared to the other cases. This experimental condition provided no predictive information (FPP or FTP) or (ground) textures. The absence of this additional information may have caused the pilot to initialize the flare at lower height. Fortunately, pilots generally compensated for this late flare initiation by pulling back the control column faster (see Fig. 18) to reduce the sink rate at touchdown to a level that was comparable with the other experimental conditions that did not provide outside visibility. Still, this indicates that a FPV by itself did not provide enough visual information for conducting a flare maneuver and augmentation is preferred.

This experiment indicated significant performance differences between manual landings that are executed with and without available outside vision. When considering manual performed landings with the synthetic vision display as only useful source of visual information (no available outside visibility) it was noted that the addition of predictive landing guidance supports pilots in performing landings under these conditions. Also, the additional information provided by the addition of (ground) textures to the synthetic vision display supported pilots in a similar way. Still, the provided support by augmenting the display with predictive guidance or by the addition of (ground) textures was not effective to the extent that a similar performance level was achieved relative to the condition with outside visual. Pilots appeared to perform better timed and controlled flare maneuvers, although the main performance measures (consumed runway distance and especially sink rate at touchdown) did not improve and were, overall, rather low.

\section{CONCLUSIONS}

Although the experiment indicates that it is possible to manually land an aircraft without available outside visibility and using synthetic vision, the vertical velocity at touchdown was found to be too high. With regard to the flare maneuver, which ultimately determines the performance during the landing, the addition of predictive guidance cues did enhance the pilot's ability to determine the correct flare initiation time instance somewhat. The same effect was noticed when more pictorial detail is displayed by rendering (ground) textures on the synthetic vision display.

Although the assessment of the correct flare initiation time could be supported by augmenting the synthetic vision display with predictive guidance, the flare control after initiation was not sufficiently supported and as a result no performance benefits were found. The rather mediocre landing performance found with any of the artificial visual displays warrants future research on this topic. As more and more synthetic vision systems find their way to the general aviation cockpit, it is recommended to continue investigating the landing performance with these instruments. 
A recommendation that follows from this experiment would be the application of synthetic vision displays with a more conformal Field Of View (FOV). The FOV was chosen larger than conformal based on previous research, but a FOV on the display that mimics the view when one would be looking through a window of the same size and location may increase performance. More research is recommended on the application of head-up-displays while landing during low visibility conditions.

\section{APPENDIX: DETERMINING THE MOMENT OF FLARE INITIATION}

The final descent consists of a straight approach along the glide-path. The pilot continuously controls the aircraft, correcting for small perturbations and deviations from the desired flight path using small control inputs. At an altitude of approximately 50-60 feet, the flare is initialized by gradually pulling back the control column to increase the elevator deflection and excite the aircraft phugoid eigenmotion. This change in control strategy can be used to determine the flare initiation time instance. Still, the highfrequency elevator deflections 'hides' the exact flare initiation time. Therefore, a Moving Average Filter (MAF) was applied to provide a reliable solution [22].

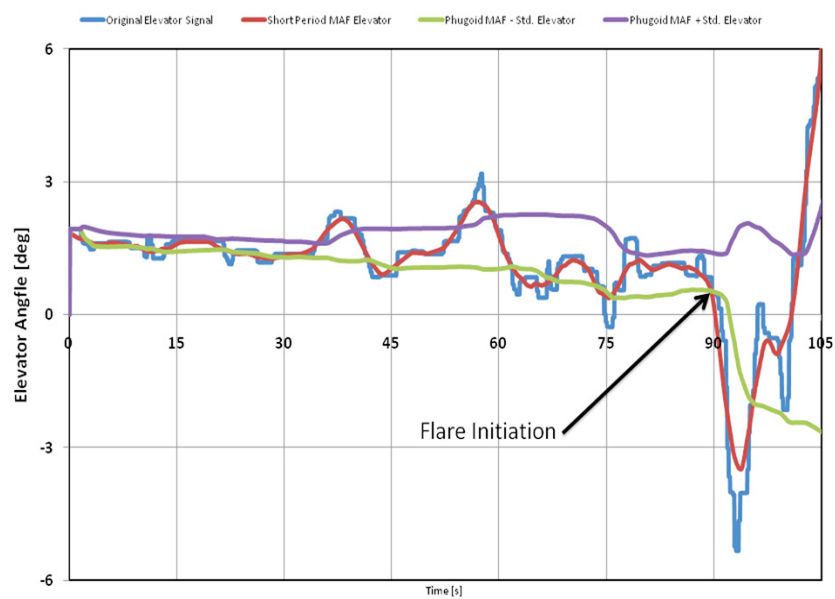

Fig. (20). Using a Moving Average Filter to determine the flare initiation time.

First, the original elevator control 'signal' is processed by a MAF with an interval length that equals the period of the short period eigenfrequency (Fig. 20). Then, the original signal is processed by a MAF with an interval length that equals the period of the phugoid and the standard deviation of the original signal within the phugoid interval is calculated. Combining the calculated standard deviation with the phugoid MAF, by subsequent addition and subtraction, establishes a deflection range. It is assumed that the control strategy is changed when the short period MAF signal exits the phugoid $\mathrm{MAF} \pm$ standard deviation range.

In Fig. (20) the signals and the phugoid MAF plus/minus one standard deviation range are illustrated. The changed control strategy that initializes the flare is indicated by the arrow. Notice that the short period MAF signal leaves the phugoid MAF \pm standard deviation range at approximately
90 seconds which indicates that the flare maneuver is initialized.

This procedure was used to determine the moment of flare initiation for all experimental runs. Visual inspections were used to verify this technique.

\section{CONFLICTS OF INTEREST STATEMENT}

The authors declare no conflict of interest.

\section{REFERENCES}

[1] A. J. Grunwald, J. B. Robertson, and J. J. Hatfield, "Experimental evaluation of a perspective tunnel display for three-dimensional helicopter approaches", Journal of Guidance and Control, vol. 4, no. 6, pp. 623-631, 1981.

[2] A. J. Grunwald, "Tunnel display for four-dimensional fixed-wing aircraft approaches, Journal of Guidance, Control, and Dynamics", vol. 7, no. 3, pp. 369-377, 1984.

[3] A. J. Grunwald. Predictor laws for pictorial flight displays, Journal of Guidance, Control, and Dynamics, vol. 8, no. 5, pp. 545-552, 1985.

[4] G. Sachs, and K. Dobler, "Predictor/Flight-Path display for manual longitudinal control improvement", Journal of Guidance, Control, and Dynamics, vol. 25, no. 3, pp. 494-501, 2002.

[5] M. Mulder, and J. A. Mulder, "A Cybernetic analysis of perspective flight-path display dimensions", Journal of Guidance, Control and Dynamics, vol. 28, no. 3, pp. 398-411, 2005.

[6] W.J.G. Pinsker, "The landing flare of large transport aircraft. Ministry of Technology: Aeronautical Research Council", London, United Kingdom, 1969.

[7] E.F. Hogge, "B-737 Linear autoland simulink model. NASA Contractor Report 2004-213021 (NASA/CR-2004-213021) ", National aeronautics and space administration NASA, Langley Research Center, Hampton, Virginia, May 2004.

[8] J.W. Rustenburg, D.O. Tipps, and D. Skinn, "A comparison of landing parameters from manual and automatic landings of airbus a-320 aircraft", Federal Aviation Administration FAA, Structural Integrity Division, Flight Integrity Group, Dayton, Ohio, UDRTM-2001-00003, 2001.

[9] E. Seckel, "The landing flare: an analysis and flight test investigation, NASA Contractor Report 2717 (NASA-CR-2517) ", National Aeronautics and Space Administration NASA, Washington, D.C., United States, May 1975.

[10] M. Mulder, J.M. Pleijsant, J.C. van der Vaart, and P.C.W. Van Wieringen, " The effects of pictorial detail on the timing of the landing flare: results of a visual simulation experiment", The International Journal of Aviation Psychology, vol. 10, no. 3, pp. 291-315, 2000.

[11] S. Palmisano, S. Favelle, W. L. Sachtler, "Effects of scenery, lighting, glideslope, and experience on timing the landing flare", Journal of Experimental Psychology: Applied, vol 14, no. 3, pp. 236-24, 2008.

[12] D.N. Lee, "Guiding Movement by Coupling Taus", Ecological Psychology, vol. 10, no. 3-4, pp. 221-250, 1998.

[13] M. Mulder, "Cybernetics of tunnel-in-the-sky displays", Ph.D. Dissertation, Faculty of Aerospace Engineering, Delft Univ. of Technology, Delft, The Netherlands, 1999.

[14] M. Jump, and G.D. Padfield, " Tau flare or not tau flare: that is the question: developing guidelines for an approach and landing sky guide. in: aiaa guidance, Navigation, and Control Conference and Exhibit", AIAA paper 2005-6404, San Francisco (CA), Aug. 15-18, 2005.

[15] G. Sachs, K. Dobler, and P. Hermle, "Flight testing synthetic vision for precise guidance close to the ground. In: AIAA Guidance, Navigation, and Control Conference and Exhibit", AIAA Paper 2004-5243, Providence (RI), Aug. 16-19, 2004.

[16] K. Alter, A. Barrows, P. Enge, C. Jennings, B. Parkinson, and J.D. Powell, "Inflight demonstrations of curved approaches and missed approaches in mountainous terrain", In: Proceedings of the ION GPS-98, Nashville (TN), September 17-20, pp. 1165-1172, 1998.

[17] G. Sachs, I. Sturhan, "Tunnel display design issues for efficient pursuit/preview control. in: AIAA guidance", Navigation, and Control Conference and Exhibit, AIAA Paper 2004-5463, Providence (RI), Aug. 16-19, 2004. 
[18] A. J. Grunwald, "Improved tunnel display for curved trajectory following: control considerations“", Journal of Guidance, Control and Dynamics, vol. 19, no. 2, pp. 370-377, 1996.

[19] A. J. Grunwald, "Improved tunnel display for curved trajectory following: experimental evaluation", Journal of Guidance Control and Dynamics, vol. 19, no. 2, pp. 378-384, 1996.

[20] S. De Stigter, M. Mulder, and M.M. Van Paassen, "On the equivalence between flight directors and flight path predictors in aircraft guidance. In: AIAA Guidance", Navigation, and Control Conference and Exhibit, AIAA paper 2005-5962, San Francisco (CA), Aug. 15-18, 2005.

[21] D.T. McRuer, and H.R. Jex, "A review of quasi-linear pilot models", IEEE Transactions on Human Factors in Electronics, vol. HFE 8, no. 3, pp. 231-249, 1967.
[22] R.R.D. Arents, "Predictive landing guidance in synthetic vision displays", MSc Thesis, NLR-TR-2006-467, National Aerospace Laboratory NLR, Amsterdam, 2007.

[23] S. G. Hart, and L. E. Staveland, "Development of NASA-TLX (task load index): results of empirical and theoretical research", In: Human Mental Workload, P., A. Hancock and N., Meshkati (Eds), Elsevier Science, North-Holland, pp. 139-183, 1988.

[24] M. W. McGreevy, and S. R. Ellis, "Direction judgement errors in perspective displays", In; Proceedings of the Twentieth Annual Conference on Manual Control. NASA Conference Publication, 2341, pp. 531-549, 1984.

(C) Arents et al.; Licensee Bentham Open.

This is an open access article licensed under the terms of the Creative Commons Attribution Non-Commercial License (http: //creativecommons.org/licenses/by$\mathrm{nc} / 3.0 /$ ) which permits unrestricted, non-commercial use, distribution and reproduction in any medium, provided the work is properly cited. 